\title{
Computer Aided Diagnosis for the Alzheimer Type Dementia
}

\author{
Ichiro Fukumoto MD. Ph.D. Prof. \\ Institute of Biomedical Engineering, Nagaoka University of Technology \\ Kamitomioka 1603-1 , Nagaoka-city , Niigata , Japan 940-2188 \\ ichiro@vos.nagaokaut.ac.jp \\ http://bio.nagaokaut.ac.jp/ fukumoto/
}

We have proposed a new dementia diagnostic method by human eye light reflexes, which demands only few seconds to measure the miotic responses and which is highly reliable as well as safe to the patients. We found that the 35 demented patients are clearly different from the 36 normal elders in the miotic parameters $(\mathrm{p}<0.01$ in Alzheimer type dementia and $\mathrm{p}<0.05$ in vascular dementia.) But the eliciting mechanisms of light reflex is not so simple and it is nor easy to understand the measured results in the clinical settings. In this study an attempt is studied to elucidate the neuro-physiological mechanism of the light reflexes with mathematical modeling and computer simulations.

The subject's eye is lightened by visible light from a small lamp in 5 seconds. The pupil images are recorded by an infrared video camera and the change of pupil diameter is analyzed by a personal computer to calculate the miotic parameters such as constriction time(s) \& constriction rate(\%). The subjects are composed of 26 normal elders, 24 Alzheimer patients (AD), 13 vascular dementia (VD) and 9 other types of dementia. The age of the 4 subject groups are all in $70 \mathrm{~s}$. The time courses of the pupil diameter in the dementia patients are clearly different from the one of the normal people as is shown in Fig.1 We have especially noticed the minimal point of the curve and have extracted the two representing parameters, namely the constriction rate and the constriction time of the minimal point. A theoretical model based on the neuro physiological knowl-

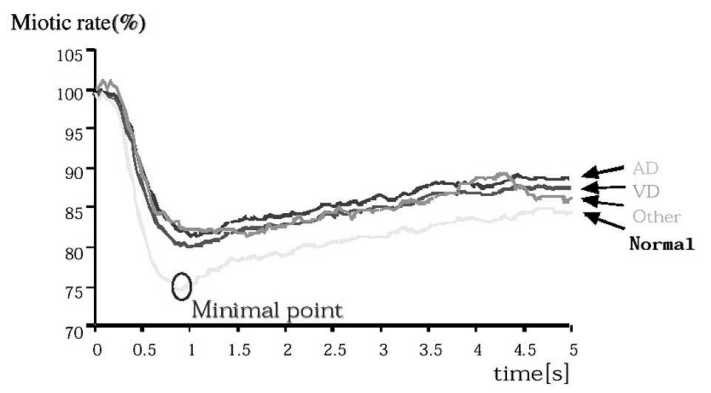

Fig. 1. The time courses of the pupil diameter in the normal and the demented people.

edge is constructed and the computer simulations using the model are executed in order to analyze the differences between the normal and the demented elders 
numerically. The model is divided into four sub-models (the primary miotic process, the secondary mydriatic process, the balancing model, modifying miotic parameter model) which reflect the neuro-physiology of the light reflex in the demented patients. In these sub-models, the decreasing activity of acetylcholine or increasing activity of adrenaline in the demented brain is considered to affect the pupil constriction and dilating force parameters namely $\mathrm{dP}, \mathrm{kP}, \mathrm{dS}, \mathrm{kS}$. The mathematical model based on the theoretical model is expressed into the three simple equations described below.

1. The activity level of the parasympathicus nerve:P(t)

(a) The delay time of the $\mathrm{P}(\mathrm{t}): \mathrm{dP}$

(b) The time constant of the $\mathrm{P}(\mathrm{t}): \mathrm{kp}$

(c) The initial value of the $\mathrm{P}(\mathrm{t}): \mathrm{P} 0$

(d) $\mathrm{P}(\mathrm{t})=\mathrm{P} 0^{*} \exp (-\mathrm{kp}(\mathrm{t}-\mathrm{dP}))$

2. The activity level of the sympathicus nerve:S(t)

(a) The delay time of the $\mathrm{S}(\mathrm{t})$ : $\mathrm{dS}$

(b) The time constant of the $\mathrm{S}(\mathrm{t}): \mathrm{kS}$

(c) The initial value of the $\mathrm{S}(\mathrm{t}): \mathrm{S} 0$

(d) $\mathrm{S}(\mathrm{t})=\mathrm{S} 0 *(1-\exp (-\mathrm{kS}(\mathrm{t}-\mathrm{dS})))$

3. A function of pupil area: $\mathrm{R}(\mathrm{t})=\mathrm{P}(\mathrm{t})+\mathrm{S}(\mathrm{t})$

Figure 2 shows an example of the simulation result (lines) as well as the measured data (circles). The results of the simulation suggest that the demented patients have the decreased activity of parasympathetic system as well as the increased activity of sympathetic system.
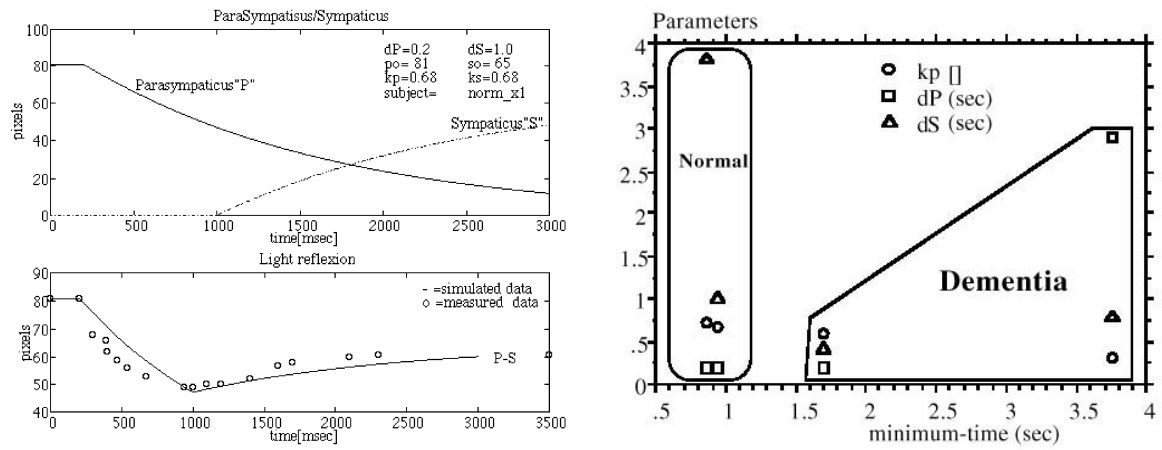

Fig. 2. The simulation result of the de- Fig. 3. The parameter analysis of the mimented elder's light reflex. otic parameters.

The miotic parameter analyses of the model using $\mathrm{kp}$, dP, dS show that the demented patients can be clearly discriminated from the normal subjects as is shown in Fig.3. It may be more easy to detect the neuro physiological changes of the demented brain, if one could adopt the neurological parameters obtained from the computer simulations on the dementia screenings, as well as the measured data from the light reflexes. The another computer simulation has been executed with good coincidences to the light reflexes too, in which the atrophic level of patients' hippocampus can be detected by MRI brain images. 